\title{
Efecto del spalling sobre la resistencia a flexión biaxial de pilares de hormigón armado expuestos a fuego
}

\section{Effect of Spalling on the Biaxial Bending Resistance of Reinforced Concrete Columns Exposed to Fire}

\author{
David L. Peña Mansilla ${ }^{\mathrm{a}}$, Vicente Albero Gabarda*,b, Carmen Ibáñez Usach ${ }^{\mathrm{a}}$, Antonio \\ Hospitaler Pérez ${ }^{\mathrm{a}}$, Ana Espinós Capilla ${ }^{\mathrm{a}}$, Manuel L. Romero García ${ }^{\mathrm{a}}$ \\ a Instituto de Ciencia y Tecnología del Hormigón. Universitat Politècnica de València. \\ ${ }^{b}$ Departamento de Ingeniería Mecánica y Construcción. Universitat Jaume I, Castellón.
}

Recibido el 30 de mayo de 2019; aceptado el 14 de diciembre de 2020

\begin{abstract}
RESUMEN
El hormigón expuesto al fuego puede sufrir spalling al aumentar la presión en los poros por evaporación del agua y esto conlleva el desconchamiento de su superficie. Este trabajo evalúa sus consecuencias sobre la resistencia a flexión biaxial de pilares de hormigón armado expuestos al fuego mediante un modelo secuencial de evaluación de la resistencia fuego. Se trata de un modelo seccional de fibras con integración por celdas. El modelo genera las superficies de interacción de los pilares a flexión biaxial y uniformemente expuestos por sus cuatro caras a la curva de fuego ISO 834.

(C)2022 Hormigón y Acero, la revista de la Asociación Española de Ingeniería Estructural (ACHE). Publicado por Cinter Divulgación Técnica S.L. Este es un artículo de acceso abierto distribuido bajo los términos de la licencia de uso Creative Commons (CC BY-NC-ND 4.0)

PALABRAS CLAVE: Hormigón armado, material, spalling, pilares, flexión, biaxial, resistencia al fuego.
\end{abstract}

\section{ABSTRACT}

Concrete exposed to fire can experience spalling when the pore pressure increases due to water evaporation and that results in the surface sloughing off. This works evaluates the consequences that spalling has on the biaxial bending resistance of reinforced concrete columns exposed to fire by means of a sequential model. In this case, it is a sectional model with fibres integration. The model is able to generate the interaction surfaces of the columns subjected to biaxial bending and 4-sided uniformly exposed to the standard fire curve ISO 834 .

(C)2022 Hormigón y Acero, the journal of the Spanish Association of Structural Engineering (ACHE). Published by Cinter Divulgación Técnica S.L. This is an open-access article distributed under the terms of the Creative Commons (CC BY-NC-ND 4.0) License

KEYWORDS: Reinforced concrete, material, spalling, columns, bending, biaxial, fire resistance.

1.

\section{INTRODUCCIÓN}

El spalling es un fenómeno que sufre el hormigón expuesto al fuego debido al aumento de presión en los poros por evaporación del agua y por el cual su capa superficial se desconcha.

* Persona de contacto / Corresponding author:

Correo-e / e-mail: valbero@uji.es (Vicente Albero Gabarda).
Este desprendimiento, violento o no, de las capas o trozos de hormigón más superficiales de un elemento estructural se da cuando, además de estar expuesto a altas temperaturas, la velocidad de calentamiento es alta, con velocidades típicamente de $20-30^{\circ} \mathrm{C} / \mathrm{min}[1]$. Cuando aparece durante los primeros 30 
minutos de exposición al fuego puede llegar a ser de carácter explosivo [2, 3]. Sin embargo, la manera en que se manifiesta a partir de los 30 minutos cambia. Así pues, el spalling puede agruparse en 4 tipologías: (a) spalling de áridos; (b) spalling explosivo; (c) spalling superficial; (d) spalling de esquina [1]. Los tres primeros están altamente influenciados por la velocidad de calentamiento, mientras que el cuarto tipo, el de esquina, depende más fuertemente de las máximas temperaturas alcanzadas y no es de tipo violento, a diferencia del resto.

El spalling produce una pérdida relevante de la sección resistente de los elementos estructurales de hormigón armado (HA). Existe un debate abierto sobre la probabilidad de aparición del spalling y las causas que lo producen. Los parámetros que influyen en la aparición del mismo son: la humedad de la masa de hormigón, la velocidad de calentamiento, estado tensional previo, la permeabilidad, la resistencia a compresión del hormigón, el recubrimiento y separación de las armaduras o el método de vibrado del hormigón [2-4].

Casi todo lo que se conoce acerca del spalling en el hormigón viene de la experiencia y observación de edificios y otras estructuras expuestas a fuego [5].

El alcance, severidad y tipo de origen es variado. El spalling puede ser insignificante en cantidad y efectos como cuando se generan únicamente hendiduras superficiales. Sin embargo, también puede tener un serio efecto en la resistencia al fuego de un elemento estructural debido al desprendimiento de una parte significante de hormigón que deje expuesto el núcleo de la sección o las armaduras, y, tras lo cual, se produzca un rápido aumento de la temperatura en estas partes, reduciendo así la respuesta de la estructura al fuego [5].

Este trabajo se centra en el estudio del spalling de esquina que ocurre en las esquinas convexas de un elemento estructura [6]. Se produce un desprendimiento de hormigón que deja expuestas las armaduras directamente a la acción del fuego [2].

El daño que el spalling causa a una estructura de hormigón armado puede hacer que los cálculos de diseño a fuego empleados ya no sean seguros y, por lo tanto, llevar a niveles bajo de seguridad al fuego [1]. Por ello, diferentes investigadores se han centrado en el desarrollo de modelos predictivos. Dwaikat y Kodur [7] implementaron un modelo unidimensional basado en el cálculo de la presión de poro. Lottman y colaboradores [8] presentaron un modelo basado en dos modelos de elemen- tos finitos acoplados donde el primero determina la evolución de la temperatura y presión de poro y, el segundo, el mecanismo de fractura que da lugar al spalling.

Sin embargo, este artículo no se centra en la predicción del fenómeno si no en evaluar las consecuencias del mismo. En el campo de las estructuras de hormigón armado, y más concretamente pilares, el reciente trabajo de Buch y Sharma [9] pone de manifiesto que todavía es materia de estudio hoy en día el efecto en la resistencia al fuego de las estructuras de cargas excéntricas en combinación con spalling. Tras llevar a cabo una campaña experimental en pilares de hormigón armado sujetos a cargas excéntricas, observan que un aumento en la excentricidad de la carga favorece la ocurrencia de spalling. Al igual que Khoury [1] concluyen que la presencia (no tanto la cuantía) de armadura longitudinal limita el spalling y que en particular son efectivas las armaduras que se distribuyen a lo largo de las caras (aunque es difícil de usar en secciones pequeñas).

En este trabajo, se pretende evaluar los efectos del spalling sobre elementos de hormigón armado, concretamente el efecto en la resistencia a flexión biaxial de pilares expuestos al fuego. Para ello se desarrolla un modelo secuencial y seccional para la evaluación de la resistencia al fuego de secciones de hormigón armado. En las secciones siguientes se describirá brevemente el modelo y su validación y, a continuación, se aplicará a la evaluación del efecto del spalling.

2.

\section{DESCRIPCIÓN Y VALIDACIÓN DEL MODELO}

En la práctica, una de las situaciones de carga más comunes para los pilares de HA es compresión combinada con flexión biaxial debida a la transferencia de carga desde las vigas y losas adyacentes. Sin embargo, la mayoría de estudios se centran en el análisis de pilares frente a compresión y flexión uniaxial.

Son escasos los modelos que se pueden encontrar en la literatura para el diseño de columnas de HA en fuego sujetas a flexión biaxial. Destacan los trabajos presentados por Wang et al. [10] y Kodur y Raut [11], quienes estudiaron numéricamente la capacidad frente al fuego de este tipo de columnas ante exposiciones asimétricas. Además, el EN 1992-1-2 [12] a)

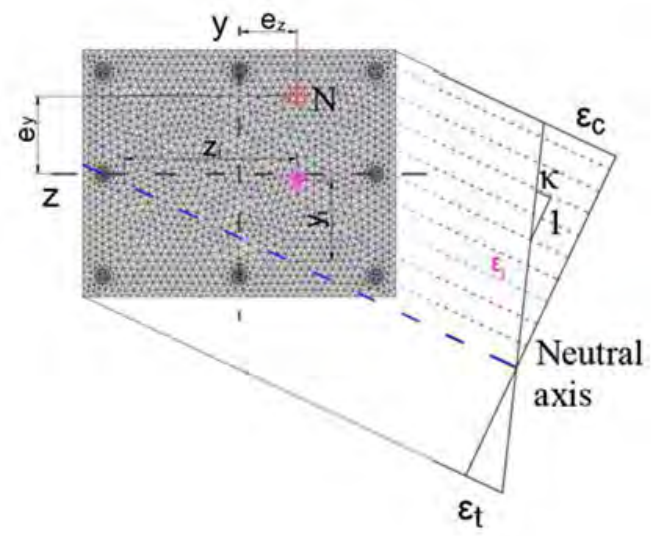

b)

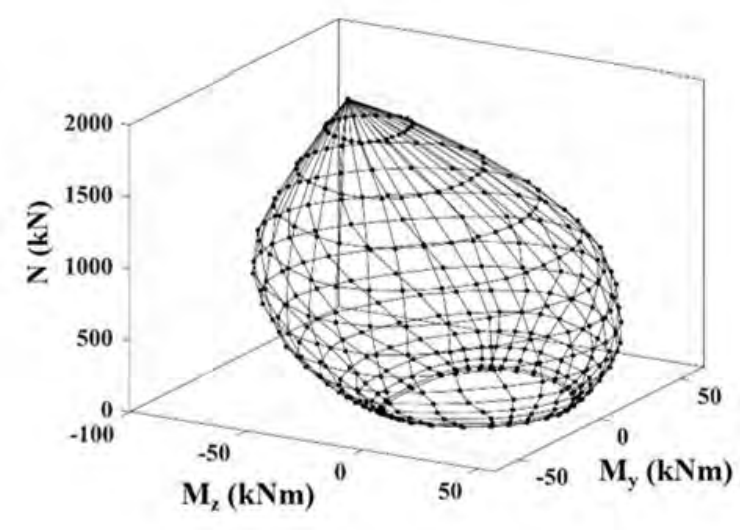

Figura 1. a) Malla seccional y distribución de deformaciones; b) Vista 3D de una superficie de interacción. 
no incluye ningún método simplificado de diseño específico. Hasta la fecha, no se han encontrado datos experimentales a altas temperaturas para pilares de HA en flexión biaxial.

Por tanto se ha desarrollado un modelo numérico con integración por fibras para la evaluación en situación de incendio de columnas de hormigón armado sujetas a flexión biaxial. Con este modelo se pueden obtener los diagramas 2D y 3D. A continuación se describe el modelo y su validación.

\subsection{Descripción}

El modelo se desarrolla en MatLab [13] y con él es posible llevar a cabo un análisis termo-mecánico secuencialmente acoplado. Este análisis consiste en dos pasos: (1) Se realiza un análisis térmico seccional para obtener el campo de temperaturas para un tiempo dado de exposición al fuego; (2) Se resuelve el problema mecánico considerando el estado térmico de la sección obtenido en el paso anterior. Para el problema de transmisión de calor se adoptan los valores recomendados en EN 1991-1-2 [14]. Para obtener las temperaturas seccionales, se aplica el método de diferencias finitas propuesto por Lie y Irwin [15].

Para el análisis mecánico, la sección se malla triangularmente (Figura la) y cada celda se caracteriza por su posición, su temperatura (mediante interpolación lineal) y por las propiedades del material. En este caso, tanto para el hormigón como para el acero, se utilizan las propiedades térmicas y mecánicas dadas por EN 1992-1-2 [12].

Se adopta la hipótesis de Navier-Bernoulli donde una sección plana permanece plana y normal al eje neutro en flexión (Figura la). La curvatura de la sección $(\varkappa)$ se obtiene de la distribución de deformaciones totales (las mecánicas son las totales menos las térmicas). El estado tensional de cada celda se obtiene con la ecuación constitutiva del material a la temperatura correspondiente. Para una carga aplicada $\mathrm{N}$ y, a través de un proceso iterativo, se actualiza la posición del eje neutro basado en el equilibrio de fuerzas de Eq. (1):

$\sum_{i=1}^{n} A_{i} \sigma_{i}-N=0$

donde $\mathrm{A}_{\mathrm{i}}$ es el área de la celda $\mathrm{i}\left(\mathrm{mm}^{2}\right), \sigma_{\mathrm{i}}$ es la tensión de la celda i $\left(\mathrm{N} / \mathrm{mm}^{2}\right)$ y $\mathrm{N}$ es la carga axial aplicada $(\mathrm{N})$.

La capacidad a flexión se obtiene tras resolver la Eq. (2) y la Eq. (3) incrementando gradualmente el valor de la curvatura seccional para generar la curva M-x:

$\sum_{i=1}^{n} A_{i} \sigma_{i} z_{i}-M_{y}=0$

$\sum_{i=1}^{n} A_{i} \sigma_{i} z_{i}-M_{z}=0$

donde $y_{i} y_{z_{i}}$ son, respectivamente, la posición en $y$ y en $z$ del centroide de la celda $\mathrm{i}(\mathrm{mm}) ; \mathrm{y} \mathrm{M}_{\mathrm{y}}$ y $\mathrm{M}_{\mathrm{z}}$ son, respectivamente, el momento aplicado en $y$ y en $z$ (Nmm).

Para obtener la superficie de interacción, el modelo genera todas las posibles situaciones de carga mediante la rotación del eje neutro y el incremento de la carga axial desde 0 hasta el valor de la resistencia plástica seccional $\left(\mathrm{N}_{\mathrm{pl}}\right)$. En la Figura $1 \mathrm{~b}$ se muestra una de estas superficies 3D para un pilar de HA expuesto por tres caras a la curva ISO 834 [14].

\subsection{Validación térmica}

El modelo térmico se valida con temperaturas experimentales de los ensayos de Lie y Irwin [15] y Kodur et al. [16]. En la validación, las predicciones para puntos internos de la sección del hormigón a diferentes tiempos de exposición al fuego se comparan con los valores experimentales. Como ejemplo, en la Figura 2 se muestra la validación para uno de los pilares. Los puntos yacen generalmente dentro de las fronteras $\pm 15 \%$. La precisión del modelo aumenta para temperaturas mayores de $400^{\circ} \mathrm{C}$. Más detalles sobre el modelo y la validación pueden encontrarse en Peña et al. [17].

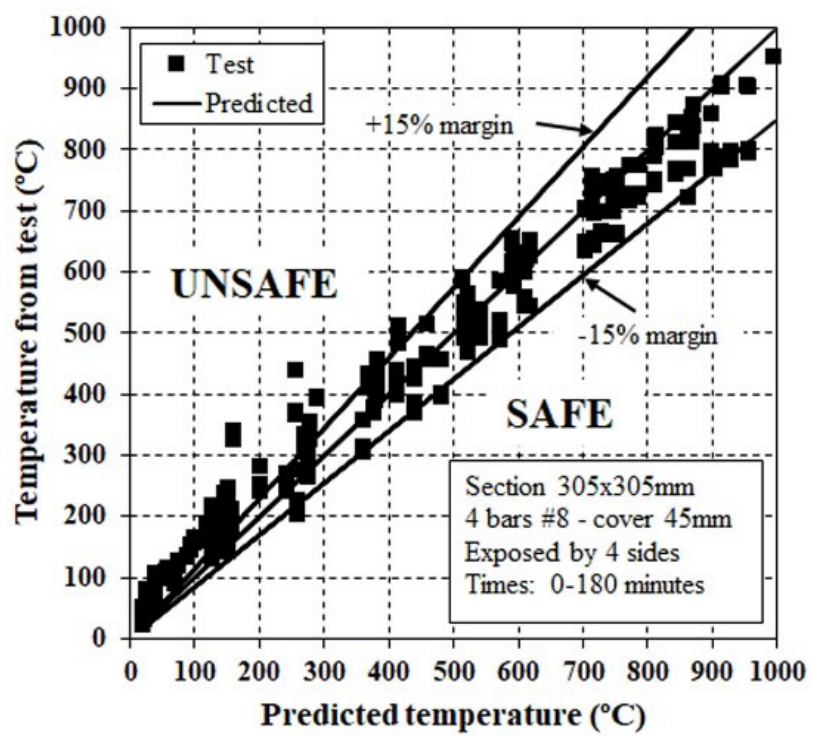

Figura 2. Tests vs. Predicción Col. 1 Lie and Irwin [15].

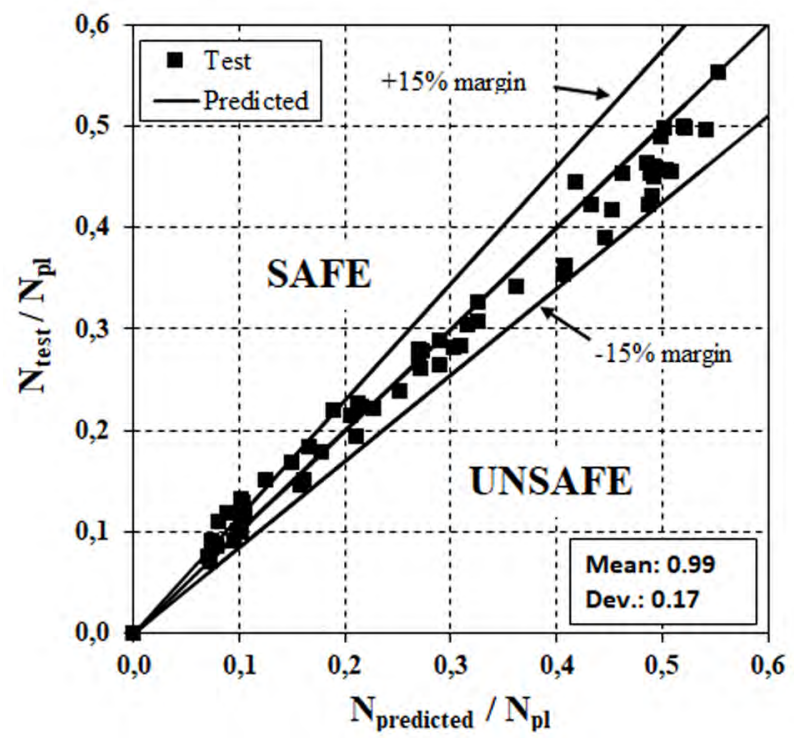

Figura 3. Tests vs. Predicciones [17].

\subsection{Validación mecánica}

Debido a la falta de experimentos en pilares a flexión biaxial expuestos a fuego, la validación del modelo mecánico se acomete en dos partes: (1) Validación a temperatura ambiente a flexión biaxial y (2) Validación a altas temperaturas bajo flexión 

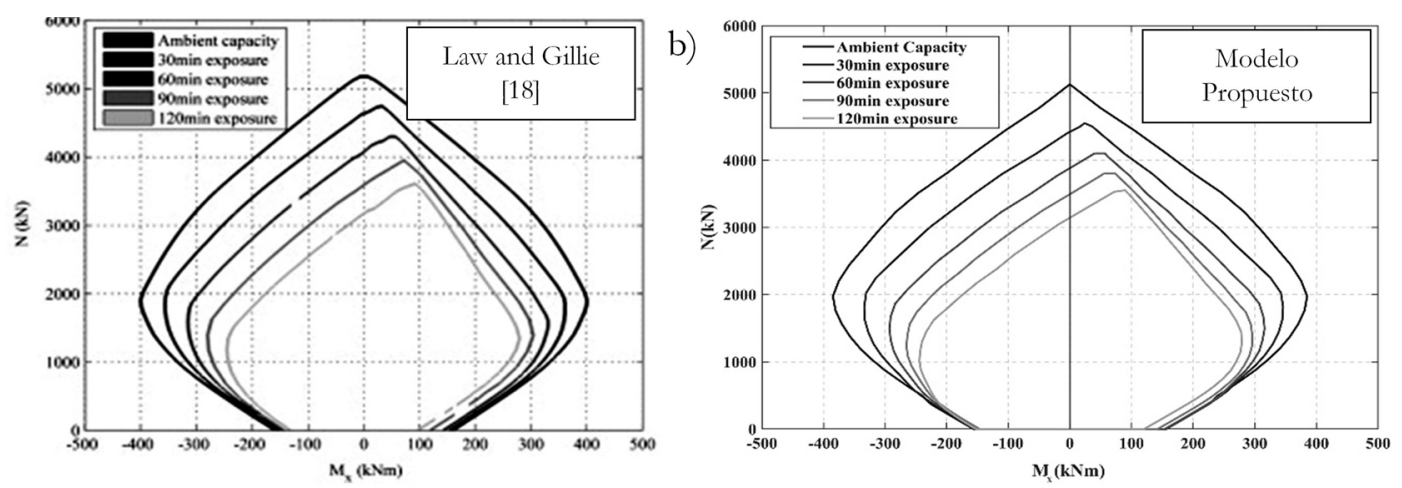

Figura 4. Diagramas de interacción uniaxiales para diferentes tiempos de exposición al fuego.

uniaxial. Para la validación a temperatura ambiente se emplean los resultados experimentales de varios estudios como se puede consultar en [17]. La Figura 3 muestra la buena correlación que existe para los 61 casos validados (Media=0.99, $\mathrm{SD}=0.17$ ).

Para la validación a altas temperaturas bajo flexión uniaxial, las predicciones del modelo se contrastan con las dadas por el modelo de Law y Gillie [18]. El pilar tomado como referencia (300x500mm, 6\$20) está expuesto a la curva ISO 834 [14] por tres lados. En la Figura 4 puede observarse como las predicciones de ambos modelos son idénticas. Por tanto, la capacidad de predicción del modelo se considera satisfactoria.

\section{3.}

\section{APLICACIÓN DEL MODELO A LA EVALUACIÓN DEL EFECTO DEL SPALLING}

En este estudio se consideran columnas de HA expuestas uniformemente por sus cuatro caras a la curva de fuego estándar. En primer lugar, se obtiene la evolución temporal del mapa térmico de la sección y cuando se produce el spalling, esto se refleja en el modelo mediante la eliminación de las fibras de hormigón afectadas (Figura 5). Los parámetros que determinan esta eliminación se fijan de acuerdo a lo concluido tras el estudio de la bibliografía más relevante en cuanto al momento en el que ocurre y profundidad de hormigón afectada. En este estudio, para esta último se ha considerado que la mitad del perímetro de la armadura de la esquina queda expuesto directamente al fuego. Por la pérdida seccional debida al spa-

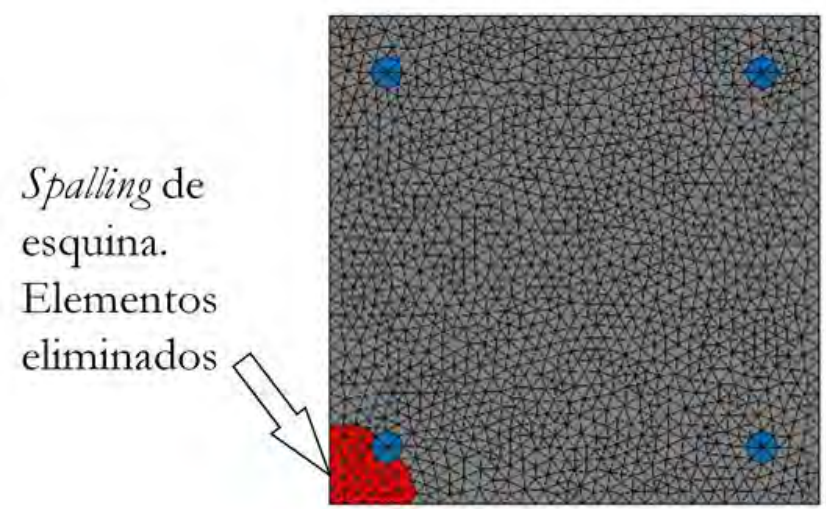

Figura 5. Modelización del spalling en esquina. lling, la simetría tanto térmica como geométrica de la sección que se tenía inicialmente se pierde. Por tanto, la obtención de temperaturas debe actualizarse para las nuevas condiciones de contorno dadas por eliminación de los elementos afectados. Además, este desprendimiento del hormigón deja expuesta la armadura. La nueva asimetría geométrica de la sección por la aparición del spalling obliga a la resolución, en cualquier caso, de un problema de flexión biaxial. Así, el modelo secuencial se encarga de obtener el mapa térmico seccional de la sección con spalling y, a continuación, su nueva respuesta mecánica.

Para realizar el estudio se toma como referencia una sección de HA-30 de 300x300 mm con un armado de $4 \Phi 16$ y con recubrimiento geométrico de $35 \mathrm{~mm}$. El parámetro que se varía es el tiempo de ocurrencia del spalling. Los tiempos evaluados son de 10, 15, 20 y 30 min desde el inicio del calentamiento, que se produce uniformemente por las cuatro caras y sigue la curva ISO 834 [14]. En la Figura 6 se muestra el mapa térmico seccional a los 30, 60 y 90 min de exposición al fuego cuando se ha producido spalling de esquina.

Se observa como en la esquina donde ocurre el spalling, la armadura queda expuesta y se calienta mucho más rápidamente que en las otras tres esquinas, como era de esperar.

En la gráfica de la Figura 7 se presenta la evolución de la temperatura en la armadura directamente expuesta al fuego por el spalling para diferentes tiempos de iniciación del mismo (10, 20 y $30 \mathrm{~min})$. Se observa claramente un aumento de la velocidad de calentamiento en el momento en el que ocurre el spalling. Sin embargo, el efecto del tiempo de iniciación es menos notable a medida que aumenta el tiempo de exposición al fuego, la diferencia de las temperaturas entre las curvas con spalling es cada vez menor y, sobre los 45 minutos, las tres curvas se superponen.

Esto pone de manifiesto que para este caso, al menos que se evalúe una resistencia al fuego menor de $45 \mathrm{~min}$, el tiempo de iniciación no tiene influencia.

Este resultado se corrobora al observar la respuesta mecánica de la sección de HA para una R60 considerando los diferentes tiempos de iniciación del spalling. En la Figura 8 se muestra las diferentes superficies de interacción y se observa como todas ellas se superponen, lo que implica la misma capacidad seccional para todos los casos estudiados.

A partir de las superficies de interacción, que se pueden obtener para cualquier tiempo de resistencia al fuego, es posible extraer los diagramas de interacción $\mathrm{M}_{\mathrm{y}} \mathrm{y}_{\mathrm{z}}$ para el nivel de carga deseado, cuyo axial asociado viene dado por la Eq. (4): 

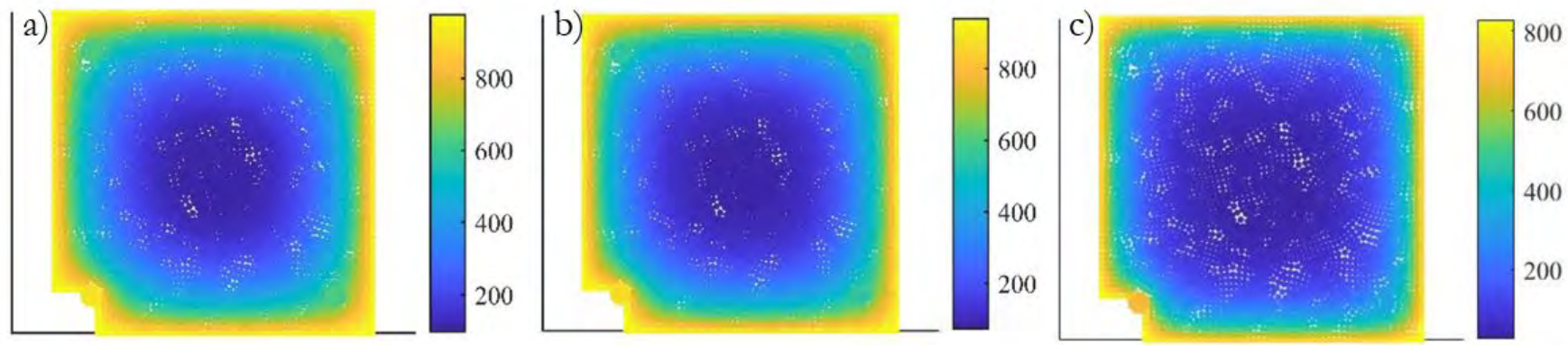

Figura 6. Mapas térmicos seccionales: a) $30 \mathrm{~min}:$ b) $60 \mathrm{~min}$; c) $90 \mathrm{~min}$.

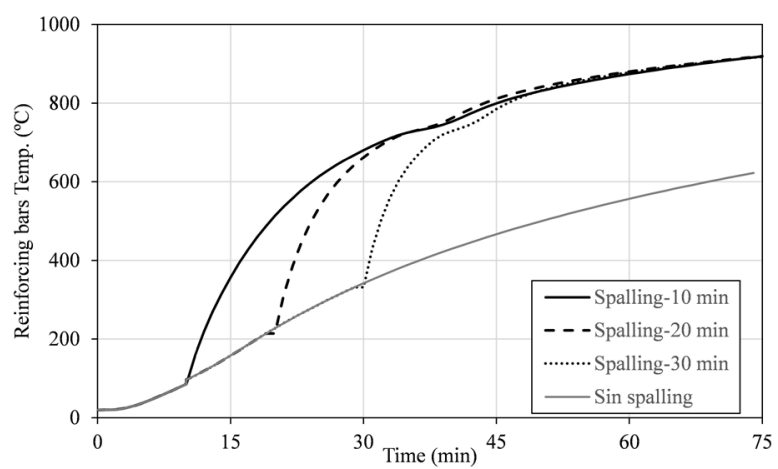

Figura 7. Temperatura de la armadura expuesta.

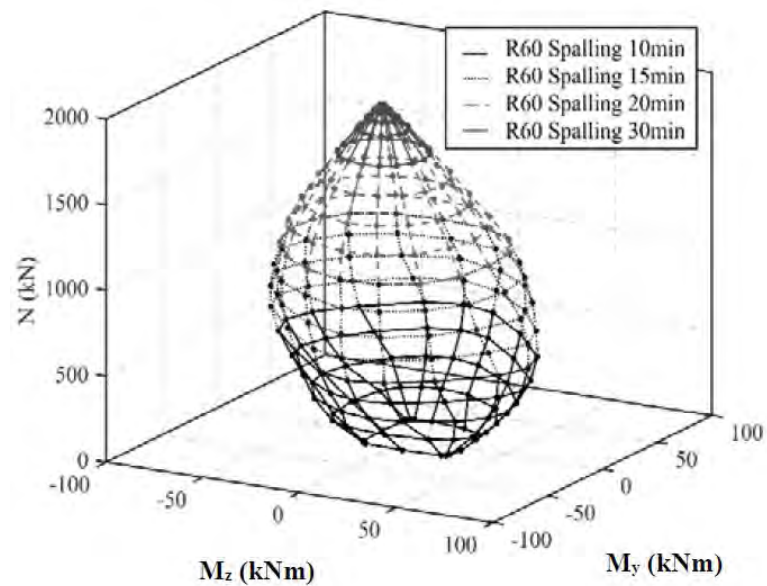

Figura 8. Superposición de las superficies de interacción para R60.

$N=\mu b h f_{c k}$

donde $N$ es la carga axial $(N)$; $\mu$ es el nivel de carga, $b$ es el ancho de la sección $(\mathrm{mm}), h$ es el canto de la sección $(\mathrm{mm})$ y $f_{c k}$ es la resistencia característica a compresión del hormigón (MPa).

Así pues, para un mismo nivel de carga, esta representación permite comparar los diagramas de interacción de la misma sección para situaciones con y sin spalling. De este modo, se puede evaluar la pérdida de resistencia al fuego que genera este fenómeno al encontrar el diagrama de interacción de la sección sin spalling que coincide, en el cuadrante más desfavorable, con la envolvente del diagrama de interacción de la sección cuando esta sí que ha sufrido spalling en su calentamiento.

Por ejemplo, en la Figura 9, se muestran en línea discontinúa el diagrama de interacción para una R30 de una sección donde ha habido. Este diagrama se obtiene al realizar un corte en la superficie de interacción 3D a la altura del axil correspondiente a un nivel de carga de 0.4 (en este caso, $1080 \mathrm{kN}$ ). En el mismo gráfico, en línea continua está grafiado el diagrama de interacción de la misma sección pero sin ocurrencia de spalling para el mismo nivel de carga y para un R50. Al no haber spalling, se observa que este diagrama es simétrico.

Como se puede observar, en el primer cuadrante, ambos diagramas coinciden en la zona más desfavorable del diagrama de R30 con spalling. Esto quiere decir que la situación más perjudicial en cuanto a comportamiento mecánico seccional para un R30 con spalling equivale a la resistencia mecánica en situación de incendio que se tendría tras 50 min de exposición (R50) si no produjera dicho fenómeno. Por tanto, el efecto del spalling equivale a prolongar el calentamiento de la sección durante 20 min más.

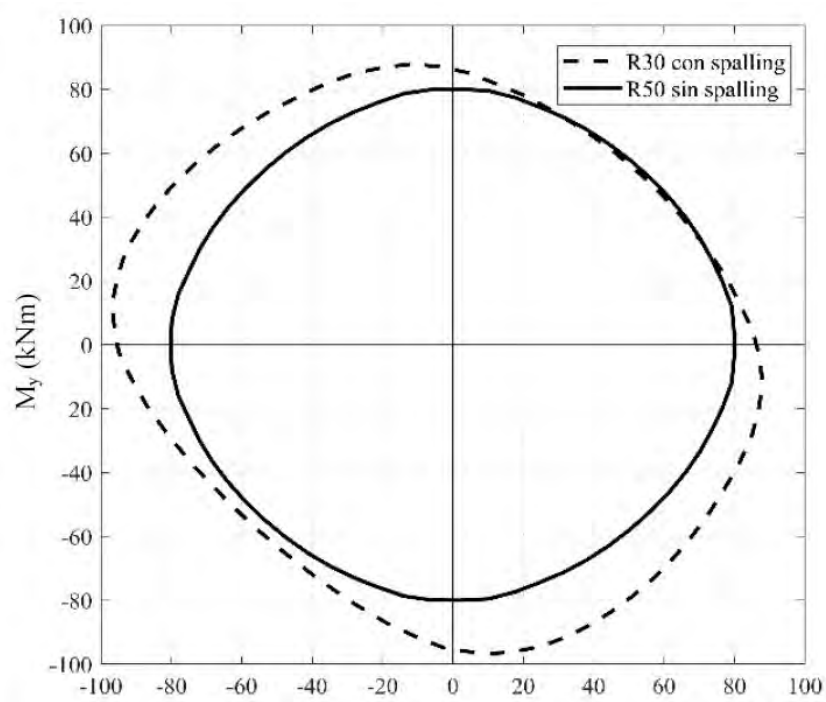

Figura 9. Diagrama de interacción R30 $\mu=0.4$.

Otro ejemplo del mismo tipo se presenta en la Figura 10. En este caso la línea discontinua es el diagrama de interacción tras 60 minutos de exposición al fuego (R60) en la sección de HA con ocurrencia de spalling. En discontinuo se muestra la capacidad mecánica que tendría la sección si no sufriese spalling tras 80 min de exposición al fuego (R80). Del mismo modo que antes, se observa que en el cuadrante más desfavorable para R60, ambos diagramas coinciden en el punto más crítico.

Estas observaciones indican que, al menos hasta tiempos de exposición al fuego de $60 \mathrm{~min}$, el efecto negativo de la ocu- 


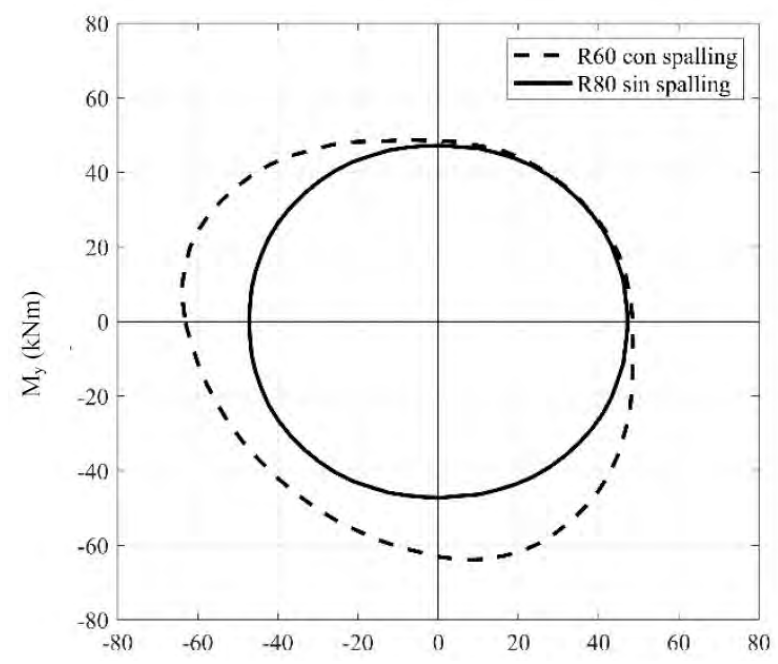

Figura 10. Diagrama de interacción $\mathrm{R} 60 \mu=0.4$.

rrencia del spalling equivale a una prolongación de 20 min en el tiempo de exposición al fuego de la sección de HA calentada uniformemente por sus cuatro caras y sin la ocurrencia de spalling.

\section{4.}

\section{CONCLUSIONES}

En este artículo se ha presentado un modelo que permite obtener la capacidad mecánica seccional de pilares de HA en situación de incendio y sujetos a flexión biaxial en los que durante el calentamiento ocurre spalling en una de sus esquinas.

Del presente trabajo, estas son las conclusiones que se pueden extraer:

- El modelo secuencialmente acoplado es capaz de generar las superficies de interacción que representan la capacidad mecánica de secciones de HA bajo flexión biaxial y ante la ocurrencia de spalling.

- Ante un spalling de esquina, la temperatura de la armadura expuesta crece mucho más rápidamente que cuando mantiene el recubrimiento.

- Tanto la capacidad mecánica de la sección como la temperatura en la armadura expuesta son sensibles al tiempo de iniciación del spalling al principio del calentamiento. A partir de los $45 \mathrm{~min}$, este parámetro no influye.

- Para un nivel de carga dado y un tiempo de exposición al fuego de una sección con spalling, es posible encontrar el diagrama de la sección sin spalling que es equivalente en el cuadrante más desfavorable.

- El efecto negativo del spalling en una sección de HA equivale a prolongar $20 \mathrm{~min}$ el calentamiento de una sección sin spalling bajo la curva ISO 834

\section{Agradecimientos}

Los autores quieren agradecer los fondos recibidos por the $\mathrm{Na}$ tional Agency for Research and Development (ANID)/Scholarship Program/DOCOTRADO BECAS CHILE/2018.(R)72190104, para el desarrollo de la investigación del primer autor en la Universitat Politècnica de València.

\section{Referencias}

[1] G.A. Khoury, Effect of fire on concrete and concrete structures. Progress in Structural Engineering and Materials. 2 (2000) 429-447

[2] J.C. Dotreppe, J.M. Franssen, Y. Vanderzeipen,Calculation Method for Design of Reinforced Concrete Columns under Fire Conditions. American Concrete Institute. 96(1) (1999) 9-18.

[3] A.M. Gil, B. Fernandes, F.L. Bolina, B.F. Tutikian, Experimental analysis of the spalling phenomenon in precast reinforced concrete columns exposed to high temperatures. Revista IBRACON de Estruturas e Materiais., 11(4) (2018) 856-875.

[4] R. Jansson, Fire spalling of concrete - A historical overview, MATEC Web of Conferences. (2013) 6, 01001.

[5] CEB-FIB: Fire design of concrete structures- materials, structures and modelling, State-of- the-art, Bulletin 38. International Federation for Structural Concrete (fib) (2007)

[6] K.D. Hertz, Limits of spalling of fire-exposed concrete. Fire Safety Journal 38 (2003) 103- 116

[7] M.B. Dwaikat, V. Kodur, Hydrothermal model for predicting fire-induced spalling in concrete structural systems. Fire safety Journal. 44(3) (2009) 425-434.

[8] B.B.G. Lottman, E.A.B. Koenders, C.B.M . Blom,.J.C. Walraven, Spalling of concrete due to fire exposure: A coupled fracture mechanics and pore pressure approach. MATEC Web of Conferences (2013) 6, 05002.

[9] S.H. Buch, U. K. Sharma, Fire resistance of eccentrically loaded reinforced concrete columns. Fire Technology 55(2019) 1517- 1552

[10] L. Wang, R. Van Coile, R. Caspeele, L. Taerwe, Simplified method for evaluating the biaxial capacity of rectangular reinforced concrete columns during fire, Materials and Structures, 50 (2017) 1-13.

[11] V. Kodur, N. Raut, A simplified approach for predicting fire resistance of reinforced concrete columns under biaxial bending, Engineering Structures, 41 (2012) 428-443.

[12] CEN., 2004b. EN 1992-1-2, Eurocode 2: Design of concrete structures, Part 1.2: General rules - Structural fire design, Brussels, Belgium: Comité Européen de Normalisation.

[13] MATLAB, 2018. Natick: The MathWorks Inc.

[14] CEN., 2002. EN 1991-1-2, Eurocode 1: Actions on structures,Part 1.2 Actions on structures exposed to fire, Brussels, Belgium: Comité Européen de Normalisation.

[15] T.T. Lie, R.J. Irwin, Evaluation of the fire resistance of reinforced concrete columns with rectangular cross- sections, Internal Report, Institute for Research in Construction, National Research Council of Canada (NRCC), Ottawa, Canada (1990)

[16] V. Kodur, F. Cheng, T. Wang, J. Latour, P. Leroux, Fire Resistance of High-Performance Concrete Columns, Internal Report, Institute for Research in Construction, National Research Council of Canada (NRCC), Ottawa, Canada (2001)

[17] D. Peña, V. Albero, A. Hospitaler, C. Ibañez, A. Espinos, M. L. Romero, Reinforced concrete columns exposed to fire: evaluation of the fire resistance under biaxial bending conditions, Applications of Structural Fire Engineering, (2019).

[18] A. Law, M. Gillie, Interaction diagrams for ambient and heated concrete sections, Engineering Structures, 32(6) (2010) 1641- 1649. 